\title{
UNA ENCRUCIJADA GERENCIAL: LA EDUCACION AMBIENTAL VS. EDUCACION PARA EL DESARROLLO SOSTENIBLE
}

Managemental crossroads: The environmental education versus education for sustainable development I Uma encruzilhada gerencial: a educação ambiental vs educação para o desenvolmento sustentável

Alejandro Escorihuela ${ }^{1}$; Yoleida Hernández ${ }^{2}$ y Danny Daniel López Juvinao ${ }^{3}$

\section{RESUMO}

A presença, no cenário internacional, dos termos Educação Ambiental (EA) e Educação para o Desenvolvimento Sustentável (EDS) tem criado divergencias, no que tange a enfoques, posturas teóricas e metodologias. Alguns investigadores que apoiam a EDS (González, 2006a e 2006b; Mayor, 2009; Novo, 2009) argumentam que a EA não contempla o aspecto social. $A$ incorporação de qualquer destes termos, dentro do marco jurídico, poderia afectar as decisões a tomar, quanto à Gestão Ambiental e a visão da educação neste ângulo. No presente trabalho, realizou-se uma investigação documental, com a finalidade de conhecer as diferentes posturas sobre ambos os termos e determinar qual deles prevalece na plataforma jurídica dos países da América do Sul (Venezuela e Colombia). Concluiuse que, para os referidos países, o termo mais frequente refere-se a EA, fazendose necessário repensar e reconstruir mecanismos que se ajustem às novas realidades, com vista a incorporar conceitos que beneficiem a sociedade, no futuro.

Palavras Chave: Educação Ambiental, Educação para o Desenvolvimento Sustentável, Gestão Ambiental.

Recebido: Novembro 2018

Aceitado: Dezembro 2018

\section{RESUMEN}

La presencia en el escenario internacional de los términos educación ambiental (EA) y educación para el desarrollo sostenible (EDS) ha creado divergencias respecto a enfoques, posturas teóricas y metodologías. Algunos investigadores que apoyan la EDS (González, 2006a y 2006b; Mayor, 2009; Novo, 2009) argumentan que la EA no contempla lo social. La incorporación de cualquiera de estos términos, dentro del marco jurídico, podría afectar las decisiones a tomar en cuanto a la gerencia ambiental y la visión de la educación al respecto. En el presente trabajo se realizó una investigación documental, con la finalidad de conocer las diferentes posturas

\footnotetext{
1 Profesor de la Universidad del Zulia, Cursante del Doctorado en Ciencias Gerenciales. Maracaibo, Venezuela. Correo electrónico: alejandro.escorihuela@hdes.luz.edu.ve

2 Ing. Forestal. Mgs. Gerencia de Empresa, y en Ecología Aplicada. Cursante del Doctorado en Ciencias Gerenciales. Mene Grande, Venezuela. Correo electrónico: yoleidahernandez61@gmail.com

${ }_{3}$ Profesor de La Universidad de La Guajira, Colombia. Doctor en Ciencias Gerenciales. Maicao, Colombia. Correo electrónico: dlopezi@uniguaijira.edu.co
} 
Una encrucijada gerencial: la educacion ambiental vs educacion para el desarrollo sostenible

sobre ambos términos y determinar cuál de ellos prevalece en la plataforma jurídica de dos países suramericanos (Venezuela y Colombia). Se concluye, que para ambos países el término manejado es el de EA, haciendo necesario repensar y reconstruir mecanismos que se ajusten a las nuevas realidades en función de incorporar conceptos que beneficien a la sociedad a futuro.

Palabras Clave: Educación ambiental, educación para el desarrollo sostenible, gerencia ambiental.

\begin{abstract}
International scene presence Environmental Education (EE) terms and Education for Sustainable Development (ESD) has crated divergence with respect to approach, theoretical position and methodologies. Some investigation support ESD (González, 2006a y 2006b; Mayor, 2009; Novo, 2009) and argues EE don't contemplate social stuff. Incorporation of any these terms, within the juridical framework, would decision affect made by environmental management. In the present work a documentary investigation was realized, with the purpose to know position different of both terms and to determine who of them prevails in the juridical platform in two South American countries (Venezuela and Colombia). It is concluded that for both countries the term is managed by EE, making it necessary to rethink and reconstruct mechanisms that fit the new realities in order to incorporate concepts that benefit society in the future.
\end{abstract}

Key words: Environmental Education, Education for the Sustainable Development, Environmental Management.

\title{
INTRODUCCIÓN
}

Nuevas interrogantes surgen en torno a la presencia en el escenario internacional, de dos conceptos que pudieran modificar la visión actual de la gerencia ambiental, o tal vez generar un nuevo método de administración de los recursos disponibles para solucionar la problemática ambiental con impacto en lo social, estos dos conceptos son educación ambiental (EA) y educación para el desarrollo sustentable (EDS), debido a que en cierto modo se encuentran relacionados, según la concepción con la cual se observen.

Ambos conceptos están relacionados con la forma a través de la cual se educa a los individuos acerca del cuidado del ambiente, uno mucho más tradicional, mientras que el segundo involucra elementos que se han venido estudiando en los últimos años, como el caso de preservar el ambiente para las generaciones futuras, relacionado con el termino de Desarrollo Sostenible, es decir, no solo cuidar el 
ambiente con la visión de preservarlo de cierta forma, sino también de prepararlo para su disposición y uso a futuro.

El desarrollo sostenible se basa en tres factores: sociedad, economía y medio ambiente, según Wikipedia (2017), básicamente se puede observar su conceptualización observando el esquema de los tres pilares del mismo, en el grafico a continuación:

Grafico. 1. Esquema de los 3 pilares del desarrollo sostenible

Fuente: Wikipedia (2017).

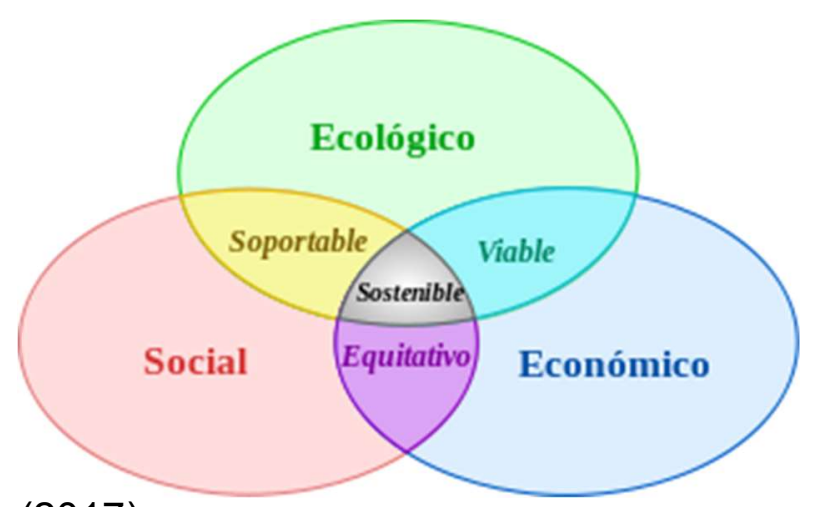

Este término, se ha vuelto famoso en los últimos años, en función de la búsqueda del beneficio a futuro de la sociedad, tomando en cuenta que el tratamiento que se le dé hoy al ambiente repercutirá en las situaciones del mismo a futuro.

El siguiente trabajo de investigación documental tiene la finalidad de conocer las diferentes posturas sobre ambos términos y determinar cuál de ellos prevalece en la plataforma jurídica de dos países suramericanos (Colombia y Venezuela) el uso de ambos términos y cual se observa mayormente dentro del marco legal vigente, para coadyuvar a unificar criterios que permitan apoyar posiciones en materia de gerencia Ambiental.

\section{CONFUSIÓN O CAMBIO}

No es de extrañar que la educación ambiental se encuentre en los actuales momentos dentro una polémica internacional, y es que desde sus inicios ha sido 
Una encrucijada gerencial: la educacion ambiental vs educacion para el desarrollo sostenible

blanco de criticas, "desde su legitimación como campo pedagógico se ha encontrado en un permanente antagonismo, o al menos divergencia, de enfoques y posturas teóricas y metodológicas" (González, 2006a:52). Sin embargo, la misma, ha sido instrumento de concientización ante los efectos observados en el medio ambiente a partir de los procesos de industrialización, cuestionando las políticas de producción que perjudiquen la sociedad, los recursos y la calidad de vida, tal y como esta establecido en su documento fundacional (Unesco, 1976).

Adicionalmente, el tratamiento de este tema en el ámbito internacional ha favorecido el "fortalecimiento ambiental del currículo en las escuelas y universidades, en la actualización docente, en materia de cultura ambiental, en la formación de tomadores de decisiones y en los procesos de desarrollo comunitario" (González, 2006a:53). No por ello, puede decirse que este Programa Internacional de Educación Ambiental (PIEA) iniciado en 1975 (Unesco, 1976), haya sido exitoso, en realidad, no ha mejorado el problema ambiental en el mundo (González, 2006a).

La dificultad del PIEA no radica en sus planteamientos teóricos, los cuales tal y como han sido concebidos pudieran haber sido de gran utilidad, "después de 30 años, sorprende la vigencia actual de los planteamientos ante la crisis ambiental, quienes construyeron el fundamento teórico, lo hicieran con tal clarividencia y con una implicación tan absolutamente comprometida en los problemas del desarrollo" (Novo, 2009, p.207).

¿Entonces si el problema no son sus fundamentos teóricos porque el PIEA no ha logrado mitigar la problemática ambiental internacional?, González (2006a) nos dice: "los cuatro millones de dólares anuales que el PNUMA canalizó a la UNESCO durante los veinte años de aplicación fueron empleados para pagar una ineficiente burocracia y un programa editorial predominante, acorde con la visión de los países desarrollados". Bajo estas circunstancias, es difícil esperar que PIEA cumpliera con uno de sus más destacados principios como la de favorecer el "desarrollo y crecimiento en una perspectiva ambiental" (Unesco, 1976) bajo mecanismos ajustados a las diferentes realidades presentes en nuestro planeta.

Así, la debilitada lucha ambiental se hacía notoria, y en 1995 aparece en el escenario internacional un nuevo concepto la educación para el desarrollo 
sostenible (EDS), impulsada por la Organización de Naciones Unidas quien designa en el 2002 a la Unesco, como órgano promotor del concepto durante 10 años, a partir del 2005 y hasta el 2014 (Calvo y Gutiérrez, 2007; Novo, 2009).

Hay quien propone que este nuevo plan educativo no es más que una medida "para recuperar la participación de los Estados Unidos dentro del organismo" (González, 2006b), y es que la disminución de los montos asignados a los proyectos eran evidente al compararlos con las dos décadas anteriores al año 1992 cuando estaba en vigencia el PIEA (González, 2006b), sea cual sea razón, desde la conferencia de Tesalónica coexisten en el panorama mundial ambos conceptos EA y EDS (Calvo \& Gutiérrez, 2007).

¿Cuál es la mejor opción?, quienes defienden el EDS argumentan que EA no contempla en su campo de acción los problemas de índole social, asunto que es totalmente falso, "La EA desde su nacimiento, ha sido un movimiento con ingerencia en los problemas sociales" (Jickling, 2006:66), es, "un instrumento de transformación social y empoderamiento de los más débiles, todo ello con la meta final de conseguir sociedades más armónicas y equitativas que permitan hacer frente a desafíos que emergen con la complejidad ambiental" (Novo, 2009).

Señala Moré (2013: 22)

de lo que se trata es de que la educación ambiental ha evolucionado, a tono con los cambios en la conciencia y el pensamiento ambiental contemporáneo; reorientando sus objetivos, de la protección del medio ambiente al tránsito hacia el desarrollo sostenible; lo que no justifica el planteamiento de que está agotada y de que se requiere de otro proceso diferente y descubierto por el Norte, que no va más allá de un cambio de nombre sin rebasar los aspectos formales.

Aparicio (2011: 105) resalta que "la Educación Ambiental es una tarea de formación no sólo información aceptando que entre los más nobles objetivos de la universidad está el de formar personas capaces de concebir fines y de actuar coherentemente con la realidad".

Por lo tanto, ¿cuál es la innovación que presenta la EDS?, concepto que además de ser poco conocido no parece estar muy claro, producto del desmesurado 
Una encrucijada gerencial: la educacion ambiental vs educacion para el desarrollo sostenible

uso con distintas definiciones (Dobson, 1996), y por ello su fracaso en todo el mundo, y muy particularmente en el llamado tercer mundo (Goñi \& Goin, 2006).

El informe Framework for the UNEDESD International Implementation Scheme (UNESCO, 2006), muestra su esquema temático: (a) Derechos humanos. (b) Paz y seguridad humanas. (c) Equidad de género. (d) Diversidad cultural y entendimiento intercultural. (e) Salud. (f) Sida. (g) Gobernanza. (h) Recursos naturales (agua, energía, agricultura, biodiversidad). (i) Cambio climático. (j) Desarrollo rural. (k) Urbanización sostenible. (I) Prevención y mitigación de desastres. (m) Reducción de la pobreza. (n) Responsabilidad social corporativa, y (p) Economía de mercado.

La Organización de Naciones Unidas a través de este paquete temático busca producir cambios dentro del panorama social mundial, al tratar de introducir la EDS en todas los sistemas de Educación (Unesco, 2009), como un órgano vinculante y transformador, "es necesario promover una cultura de la sostenibilidad, no sólo en el ámbito político, sino también en el ámbito de los agentes sociales y el conjunto de los ciudadanos" (Aznar, 2003:224).

Hay quien sugiere que la globalización puede ser un factor que incida en la determinación de un modelo educativo (Jickling, 2006), y es que la globalización nació como un arma de dominación, en "el principio de la década de los noventa, el predominio de los países más ricos impuso la globalización, con la creación y ampliación de grandes consorcios empresariales multinacionales" (Mayor, 2009:29), "es una forma de neocolonialismo más sutil y sofisticada" (Meira, 2006:111).

Por lo anteriormente expuesto, nos preguntamos ¿existen fundamentos sólidos que justifique la transición entre un concepto mal aplicado pero bien concebido (Educación ambiental, EA) y otro poco conocido y con una estructura de viabilidad dudosa (Educación para el desarrollo sustentable, EDS) promocionada por los más poderosos? Moré (2013) aclara que no se trata de pasar de un concepto a otro, ya que el concepto de educación ambiental no es estático, sino que evoluciona de forma paralela a como lo hace la concepción de medio ambiente y la percepción que se tiene del mismo. 
Moré (2013: 22-23) plantea que la EDS constituye una arista de la EA y la conceptualiza como

El proceso de educación ambiental caracterizado por la capacidad de síntesis en el estudio del medio ambiente y de los problemas de desarrollo; el estudio más profundo de las relaciones existentes entre calidad ambiental, ecología, factores socioeconómicos y tendencias políticas, mediante una visión holística de los problemas.

No se puede dejar de lado, que la gerencia ambiental que surge como una metodología "sistemática que facilite la planeación, dirección, toma de decisiones y evaluación del ambiente como un sistema susceptible de ser administrado y gestionado" (Cáceres, 2008:155), utiliza la plataforma jurídica establecida en cada país como instrumento para la toma de decisiones, en consecuencia, ¿es jurídicamente posible sostener un nuevo concepto (EDS), sin que esto impacte la visión del modelo de gestión actual?, una vista al marco legal de cada nación pudiera ayudar a resolver ese cuestionamiento.

\section{MARCO LEGAL DE LA EDUCACIÓN PARA EL DESARROLLO SOSTENIBLE Y DE LA EDUCACIÓN AMBIENTAL EN COLOMBIA}

En Colombia, según información del Ministerio del Medio Ambiente y del Ministerio de Educación Nacional (2002), los esfuerzos legislativos en materia de educación ambiental han venido acrecentando gradualmente desde diciembre de 1974, cuando entra en vigencia el código nacional de los recursos naturales renovables y de protección del medio ambiente, el cual estipula en el título II de la parte III, las disposiciones relacionadas con la educación ambiental y específicamente las disposiciones para el sector formal. Dicho código sirvió como instrumento inicial para la apertura de espacios formativos y de proyección para el manejo adecuado del ambiente en ese país.

En la Constitución de 1991 (República de Colombia, 1991), se establecen parámetros legales que posibilitan el trabajo en educación ambiental, demostrando así que el país adquiriere una conciencia más clara sobre los propósitos de manejo del ambiente y de promoción de una cultura responsable y ética al respecto; es así 
Una encrucijada gerencial: la educacion ambiental vs educacion para el desarrollo sostenible

como, la procuraduría y la contraloría deben velar por la conservación, la protección y la promoción de un ambiente sano.

En ese orden de ideas, Bermúdez (2001) señala que la educación ambiental se concibe como un espacio de análisis y reflexión, que amerita avanzar en su elaboración conceptual para propiciar un enfoque interdisciplinario de intercambio de ideas y la visión compleja en torno a lo ambiental; desde esta perspectiva, se pretende suscitar en los estudiantes el análisis y reflexión en torno a los problemas ambientales más relevantes de nuestra sociedad y motivar la búsqueda de alternativas de solución a la crisis ambiental por medio de la gestación de trabajos de investigación.

Por otro lado, la Ley 99 de 1993 (República de Colombia, 1993) por medio de la cual se crea el Ministerio del Medio Ambiente, establece en su marco el mecanismo de concertación con el Ministerio de Educación Nacional, para la adopción conjunta de programas, planes de estudio y propuestas curriculares en materia de Educación Ambiental, y así, lograr los impactos requeridos en lo que a la construcción de una cultura ambiental se refiere. Por su parte, la Ley 70 de 1993, incorpora en varios de sus artículos la dimensión ambiental dentro de los programas de etnoeducación, dirigidos a las comunidades afrocolombianas que habitan los territorios aledaños al mar Pacífico, elemento este importante para la proyección de las políticas nacionales educativas y ambientales y su contextualización en el marco de la diversidad cultural y atendiendo a las cosmovisiones propias del carácter pluricultural del país.

En ese sentido, de acuerdo al Ministerio de Educación Nacional (2009) educar para el desarrollo sostenible implica la importancia de las temáticas transversales para la mejora de la calidad educativa, es decir, la dimensión ética, cultural y científica de la educación ambiental, de modo que se establezca una política nacional que consolide líneas de acción en pro de una educación para un ambiente sostenible; entonces, la Educación Ambiental se posiciona como la instancia que permite una construcción colectiva de nuevos valores y garantiza un cambio a largo plazo, un proceso de sensibilización, concientización y participación y propende que el hombre mejore su actuación sobre la naturaleza, haciendo un 
aprovechamiento sostenible de los recursos de que dispone y a los cuales tiene acceso.

De esta forma, en la Política nacional de Colombia enunciada en el Sistema Nacional Ambiental (SINA) el termino Educación ambiental prevalece históricamente en el marco legal del país y sus objetivos están dirigidos a: (a) Difundir valores de respeto a todas las formas de vida, contribuir a la convivencia y participación ciudadanas para lograr una gestión racional de los recursos con previsión hacia el futuro; (b) Impulsar la investigación, con énfasis en la identificación y evaluación de problemas actuales, para propiciar la construcción de modelos económicos y sociales ambientalmente sustentables; (c) Contribuir al cambio cultural desarrollando modelos educativos propios, acordes con las necesidades dimensión ambiental desde una visión interdisciplinaria, holística y compleja.

Desde 1991 Colombia ha venido desarrollando una propuesta nacional de Educación Ambiental, cuyos esfuerzos fundamentales han estado orientados a la inclusión de la temática, tanto en el sector ambiental como en el sector educativo específicamente. En el sector educativo, la Educación Ambiental se ha venido incluyendo como una de las estrategias importantes de las políticas, en el marco de la reforma educativa nacional y desde los conceptos de autonomía y descentralización. En el contexto anterior, se han logrado avances significativos en lo que tiene que ver con el proceso de institucionalización, tanto a nivel nacional como a nivel regional o local.

La construcción de la propuesta de Educación Ambiental en el currículo, ha estado acompañada permanentemente de una concepción investigativa, relacionada en forma directa con la orientación que se la ha dado al mencionado programa. Esta concepción tiene fundamentos en la reflexión crítica, en una visión integradora y de proyección a la resolución de problemas, en un trabajo permanente de análisis y síntesis de la lectura de contextos y en la construcción de explicaciones para la comprensión de problemas ambientales (Ministerio del Medio Ambiente y Ministerio de Educación Nacional, 2002). 
Una encrucijada gerencial: la educacion ambiental vs educacion para el desarrollo sostenible

De esta manera, la inclusión de la Educación Ambiental en el currículo no se hace ni a través de una materia más, ni a través de una cátedra, ni a través de una disciplina o de acciones aisladas, no inscritas dentro de un proceso secuencial y permanente de formación. La Educación Ambiental en la reforma educativa, está concebida desde la visión sistémica del ambiente, desde la investigación pedagógica y didáctica para el tratamiento de problemas de diagnóstico ambiental particular y desde la idea de formación de dinamizadores ambientales, en el marco de procesos de cualificación conceptuales, metodológicos y estratégicos (Ministerio del Medio Ambiente y Ministerio de Educación Nacional, 2002). En términos generales, la propuesta se puede plantear desde los siguientes tópicos: a) Trabajo por problema ambiental, a través de proyectos ambientales escolares (PRAES); b) Construcción de escuela abierta, con proyección comunitaria; c) Formación permanente de maestros y dinamizadores ambientales a través de la investigación; d) Construcción de currículos flexibles; e) Formación para el trabajo intersectorial, interinstitucional e intercultural; f) Formación para el trabajo interdisciplinario; y, g) Formación para el reconocimiento de género.

Igualmente, el tipo de escuela que se busca, obliga a volver la mirada sobre un currículo flexible, en el que las fronteras disciplinarias no sean obstáculo para el quehacer del maestro y no limiten su papel con los alumnos y con su comunidad y en el que se refleje la diversidad natural, social y cultural. El currículo debe estar ligado a la cotidianidad de los alumnos y propiciar situaciones de aprendizaje conectadas con su realidad. Debe, así mismo, dar paso abiertamente a la construcción del saber y, por ende, a la construcción del mundo (Ministerio del Medio Ambiente y Ministerio de Educación Nacional, 2002).

De esta forma se observa como el término de Educación Ambiental (EA) ha sido históricamente usado en Colombia y es el que prevalece en las leyes y escritos que tratan los temas ambientales, fomentándola incluso desde la escuela. Por tanto, aunque el término ha evolucionado tomando en cuenta el desarrollo sostenible, para Colombia no ha sido necesario modificarlo. 


\section{MARCO LEGAL DE LA EDUCACIÓN PARA EL DESARROLLO SOSTENIBLE Y DE LA EDUCACIÓN AMBIENTAL EN VENEZUELA}

En su concepción más amplia, la legislación ambiental, desde el punto de vista histórico, ha surgido por la necesidad de regular las relaciones entre el hombre y el ambiente (Sosa \& Mantero, 1983). En tal sentido, en la década de los setenta comienza a sentirse la influencia de las grandes conferencias internacionales ambientales, contribuyendo en cierta medida con la elaboración y promulgación de la Ley Orgánica del Ambiente (República de Venezuela, 1976), cuyo objetivo era establecer, dentro de la política de desarrollo integral de la nación, los principios rectores de la conservación, defensa y mejoramiento del ambiente en beneficio de la calidad de la vida, y el fomento de la conciencia ambiental, entre otros aspectos.

Para la materialización de la citada Ley, en abril de 1977 entra en funcionamiento el Ministerio del Ambiente y de los Recursos Naturales Renovables (MARNR) como organismo rector de las políticas ambientales del estado y fija como objetivo general garantizar que el país logre un desarrollo sustentable a fin de alcanzar una mejor calidad de vida, mediante la conservación del patrimonio natural, la protección ambiental, la ordenación del territorio y la creación de la conciencia ambiental. Así mismo, desde sus inicios el MARNR estableció entre sus políticas el desarrollo de la educación ambiental del país (Gabaldón, 1983).

En este orden de ideas el MARNR establece una estrecha interrelación con el Ministerio de Educación, por mandato contenido en la Ley Orgánica de Educación decretada en 1980 (República de Venezuela, 1980), donde señala en su Artículo 3 que la educación fomentará el desarrollo de una conciencia ciudadana para la conservación, defensa y mejoramiento del ambiente, calidad de vida y el uso racional de los recursos naturales. Dichos ministerios, concretan su relación mediante la creación del Concejo Nacional para la Educación Ambiental

A finales de la década de los noventas hasta los actuales momentos, se han promulgado un conjunto de normas jurídicas en cuestiones ambientales, que de alguna manera está relacionada con la educación ambiental. En tal sentido, en el 2000 fue aprobada la nueva Constitución de la República Bolivariana (Asamblea Nacional Constituyente, 2000), el cual establece en su Capitulo IX los derechos 
Una encrucijada gerencial: la educacion ambiental vs educacion para el desarrollo sostenible

ambientales de los ciudadanos del país. Además de este articulado, y como un avance de gran importancia para la promoción de la conciencia ambiental, establece en el artículo 107 que la educación ambiental es obligatoria en los niveles y modalidades del sistema educativo, como también en la educación ciudadana no formal

Así mismo, en el 2006 se publico la Ley Orgánica del Ambiente (Asamblea Nacional, 2006), donde otorga un papel importante a la Educación Ambiental, consolidado en el Capítulo I del Título IV, con miras a promover, generar, desarrollar y consolidar en los ciudadanos y ciudadanas conocimientos, aptitudes y actitudes para contribuir con la transformación de la sociedad, que se reflejará en alternativas de solución a los problemas socio - ambientales. En el 2009 se promulga la Ley Orgánica de Educación (República Bolivariana de Venezuela, 2009), en la cual contempla en el Artículo 14 que la Educación Ambiental es de obligatorio cumplimiento, en las instituciones y centros educativos oficiales y privados.

Como se puede apreciar, desde la década de los setenta, en Venezuela, se ha implantado la Educación Ambiental en el ordenamiento jurídico, bajo la responsabilidad de las Instituciones conocidas actualmente como Ministerio del Poder Popular para el Ambiente en coordinación con el Ministerio de Educación, Cultura y Deporte. Sin embargo, esta Educación Ambiental debe estar abierta a nuevos enfoques para convertirse en un proceso de formación de competencias y posibilidades para la acción ambiental, desde una perspectiva democrática y participativa basada en una ética a favor de la vida, la pluralidad, la justicia y la equidad. En lo que respecta a la Educación para el desarrollo sostenible, no se encuentra inmersa dentro del marco jurídico venezolano, y por lo tanto su aplicación no sería factible, quedando su postulado como material de referencia.

Según la Constitución de la República Bolivariana de Venezuela (Asamblea Nacional Constituyente, 2000), en su Artículo 107: La Educación Ambiental es obligatoria en los niveles y modalidades del sistema educativo, así como también en la educación ciudadana no formal.

En vista de que la Educación Ambiental no es una asignatura aislada en el currículo, sino que debe concebirse como un eje transversal, la misma debe 
considerar las costumbres y las culturas locales, vincularse a situaciones vivenciales o de coordinación con otros sectores para promover actividades a favor del medio ambiente, responder a políticas institucionales contempladas por organizaciones gubernamentales, no gubernamentales y de educación no formales (Rojas et al., 2003).

En función de ello, las prácticas pedagógicas estarán orientadas a la toma de conciencia sobre el deterioro ambiental, la protección y mejora del ambiente en zonas rurales y urbanas. Por lo antes expuesto, se consideró relevante analizar el principio de transversalidad curricular en la enseñanza de la educación ambiental en la Segunda Etapa de la Educación Básica. De esta manera se podría establecer si la educación ambiental se concibe como un eje transversal que se manifiesta en la práctica pedagógica del docente (Fuentes et al, 2006).

Como se observó en el desarrollo del apartado, en Venezuela también prevalece el termino de Educación Ambiental (EA), incluso en Venezuela las leyes han sido modificadas posteriormente que en Colombia e igual se mantuvo el termino de EA como guía para los temas ambientales.

\section{CONSIDERACIONES FINALES}

A diferencia de la Educación Ambiental para el Desarrollo Sostenible, la Educación Ambiental desde su promulgación se ha insertado en el marco jurídico de muchos países, entre ellos Colombia y Venezuela, donde los organismos rectores en materia ambiental han desarrollado políticas de gerencia ambiental para alcanzar los cambios culturales y sociales necesarios para el logro de la preservación del ambiente.

En función del objetivo propuesto se logro conocer que para ambos países, se maneja más ampliamente el termino de Educación Ambiental, mientras que la Educación para el Desarrollo Sostenible no se observa incluida en las leyes referidas al ambiente; del mismo modo en que no se observa que la gerencia ambiental este orientada al logro de un desarrollo a futuro.

Se observa como en ambos países se ha hecho un esfuerzo por ir más allá de la inclusión de la Educación Ambiental como parte del currículo, tratando de 
Una encrucijada gerencial: la educacion ambiental vs educacion para el desarrollo sostenible

incluirla en el caso de Colombia desde la visión sistémica del ambiente, desde la investigación pedagógica y didáctica para el tratamiento de problemas de diagnóstico ambiental particular; mientras que en Venezuela se maneja como eje transversal dentro del currículo, observando como de forma similar no se enuncia directamente su inclusión como una materia o cátedra, sino que se pide que la misma sea tomada en cuenta para los temas que se traten en todas las cátedras, en función de su inherencia en los mismos.

Como recomendación, debido a que la implementación de los programas de Educación Ambiental no han sido los mas acertados, se hace necesario, repensar y reconstruir mecanismos que se ajusten a las nuevas realidades (políticas, ambiental, social, cultural y económicas) y cumplir con su visión de crear conciencia para un cambio de actitud y aptitud en la sociedad, haciéndolas participe en la búsqueda de las solución de sus propios problemas y las de naturaleza ambiental con el fin de lograr un desarrollo sostenible.

\section{REFERENCIAS BIBLIOGRÁFICAS}

Aparicio, Zhenia. (2011). Promoción de la Educación Ambiental para el Desarrollo Sostenible.

Extraído

de: https://pirhua.udep.edu.pe/bitstream/handle/11042/1416/MAE EDUC 086.p df?sequence=1 consulta: 12/11/2018.

Asamblea Nacional Constituyente. (2000). Constitución de la República Bolivariana de Venezuela. Gaceta Oficial de la República de Venezuela N 5.453. Venezuela.

Asamblea Nacional. (2006). Ley orgánica del ambiente. Gaceta Oficial de la República de Venezuela $\mathrm{N}^{\circ}$ 5.833. Venezuela.

Aznar, Pilar. (2003). Participación de las agencias educativas en el desarrollo sostenible a nivel local: hacia una Agenda 21 escolar. Revista Española de Pedagogía. No. 225, España (Pp. 223-241).

Bermúdez, Olga. (2001). Educación para el Desarrollo Sostenible. Extraído de http://www.rds.org.co consulta: 03/09/2009.

Cáceres, Gladys. (2008). La gerencia ambiental como metodología integradora del conocimiento para la administración y gestión del ambiente. Fermentum. Año 18, No. 51, Venezuela (Pp. 148-173).

Calvo, Susana y Gutiérrez, José. (2007). El Espejismo de la Educación Ambiental. Morata S.L. España.

Dobson, Andrew. (1996), Environment sustainabilities: An analysis and a typology, en Environmental Politics, Vol. 5, No. 3, Reino Unido. (Pp. 401-428). 
Fuentes, Lorena; Caldera, Yaxcelis y Mendoza, Ivan. (2006). La transversalidad curricular y la enseñanza de la educación ambiental. Revista ORBIS I Ciencias Humanas. Año 2 / № 4, Venezuela. (Pp. 39-59).

Gabaldón, Arnoldo. (1983). El Ministerio del Ambiente y de los Recursos Naturales. Ediciones de la Presidencia de la República. Venezuela

González, Edgar. (2006a). Configuración y significado Educación para el desarrollo sustentable, Trayectorias. Vol. VIII, No. 20-21, México. (Pp. 100-109).

González, Edgar. (2006b). Campo de Partida: Educación Ambiental y Educación para el Desarrollo Sustentable: ¿Tensión o transición? Trayectorias. Vol. VIII, No. 20-21, México. (Pp. 52-62).

Goñi, Ricardo y Goin, F. J. (2006). Marco Conceptual para la Definición del Desarrollo Sustentable. Salud Colectiva, Vol. 2 No. 2, Argentina. (Pp. 191198).

Jickling, Bob. (2006). Advertencia sostenida: Desarrollo sustentable en un mundo globalizador. Trayectorias, Vol. VIII, No. 20-21, México. (Pp. 63-73).

Mayor, Federico. (2009). La problemática de la sostenibilidad en un mundo globalizado. Revista de Educación, número extraordinario 2009, España (Pp. 25-52).

Meira, Pablo. (2006), In praise of environmentaleducation, en Policy Futures in Education, vol. 3, núm. 3. Extraído de: https://www.researchgate.net/publication/274599619 In Praise of Environ mental Education consulta: 03/09/2018.

Ministerio de Educación Nacional (2009). Educar para el desarrollo sostenible. Extraído de https://www.mineducacion.gov.co/1621/article-90893.html consulta: 09/09/2018.

Ministerio del Medio Ambiente y Ministerio de Educación Nacional (2002). Política nacional de educación ambiental - SINA. Colombia. Extraído de http://cmap.upb.edu.co/rid=1195259861703 152904399 919/politica educacion amb.pdf consulta: 09/09/2018.

Moré, Mavel. (2013) la educación ambiental para el desarrollo sostenible en la didáctica de las ciencias de la naturaleza, educación primaria. Extraído de: http://www.redalyc.org/html/4780/478048960002/ consulta: 12/11/2018.

Novo, María. (2009). La Educación ambiental, una Genuina Educación Para el Desarrollo Sostenible. Revista de Educación. Número extraordinario 2009, España. (Pp. 195-217).

República Bolivariana de Venezuela (2009). Ley orgánica de la educación. Gaceta Oficial de la República de Venezuela $N^{\circ}$ 5.929. Venezuela.

República de Colombia. (1991). Constitución política de Colombia. Gaceta Constitucional No. 116 de 20 de julio de 1991. Colombia.

República de Colombia. (1993). Creación del Ministerio del Medio Ambiente. Diario oficial 41.146 del 22 de diciembre de 1993. Colombia.

República de Venezuela (1980). Ley orgánica de educación. Gaceta oficial de la república de Venezuela $\mathrm{N}^{\circ} 2.635$. Venezuela. 
Una encrucijada gerencial: la educacion ambiental vs educacion para el desarrollo sostenible

República de Venezuela. (1976). Ley Orgánica del Ambiente Gaceta Oficial de la República de Venezuela $N^{\circ} 31.004$. Venezuela.

Rojas, Eduardo, Quintero, Josefina y Ancízar, Raúl. (2003). Investigación pedagógica en el currículo de educación ambiental en la Universidad de Caldas Colombia. Revista Iberoamericana de Educación, No. 11. España (Pp. 1-12).

Sosa, Cecilia y Mantero, Osvaldo. (1983). Derecho Ambiental Venezolano. Fundación Polar - Universidad Católica Andrés Bello. Venezuela

UNESCO (1976). Seminario Internacional de Educación Ambiental. Informe final. Doc. ED-76/WS/95. UNESCO. Francia.

UNESCO (2006). Framework for the UNDESD International Implementation Scheme. ED/DESD/2006/PI/1. UNESCO. Francia.

UNESCO (2009). Educación para el Desarrollo Sostenible. Objetivos. Extraído en Septiembre, 2009

de, http://www.unesco.org/es/education ar/themes/improving-educationquality/education-for-sustainable-development/decade-of-esd/

Wikipedia. (2017). Desarrollo Sostenible. Extraído en Marzo, 2017 de https://es.wikipedia.org/wiki/Desarrollo sostenible 